\title{
Gonadotropin-releasing hormone antagonist versus progestin for the prevention of premature luteinising hormone surges in poor responders
} undergoing in vitro fertilisation treatment: study protocol for a randomised controlled trial

Yun Wang, Yanping Kuang, Qiuju Chen ${ }^{*}$ and Renfei Cai

\begin{abstract}
Background: Progress in vitrification techniques has allowed reproductive physicians to consider new strategies for using progestin as an alternative to a GnRH analogue to improve in vitro fertilisation (IVF). However, the role of progestin in blocking luteinising hormone $(\mathrm{LH})$ surges and its potential in clinical practice are unclear, especially for poor responders. We designed a prospective randomised controlled trial (RCT) to compare the efficacy of a gonadotropin-releasing hormone $(\mathrm{GnRH})$ antagonist and progestin in blocking $\mathrm{LH}$ surges and premature ovulation in poor responders.

Methods/design: Poor responders who meet the Bologna criteria will be randomised to one of two stimulation regimens - gonadotropin-releasing hormone $(\mathrm{GnRH})$ antagonist or progestin-primed ovarian stimulation (PPOS) — using a computer-generated random number. Fresh embryos were transferred in the GnRH antagonist group and frozen embryos were transferred in the PPOS group. The primary outcome is the incidence of premature LH surges. Secondary outcomes include the number of oocytes retrieved, the number of embryos available for transfer, implantation rates and clinical pregnancy. The sample size for this trial is estimated as 340 participants, with 170 participants in each group. The data analysis will be by intention to treat.
\end{abstract}

Discussion: To our knowledge, this is the first RCT to examine the efficacy of administering progestin orally to block $\mathrm{LH}$ surges and premature ovulation compared with the $\mathrm{GnRH}$ antagonist protocols in poor responders undergoing IVF treatment.

Trial registration: www.chictr.org.cn. ChiCTR-IPR-17010906. Registered on 18 March 2017.

Keywords: GnRH antagonist, Progestin, Poor responders, LH surge

\footnotetext{
* Correspondence: chenqj75@126.com; cairenfei070@sina.com

Department of Assisted Reproduction, Shanghai Ninth People's Hospital,

Shanghai Jiaotong University School of Medicine, Zhizaoju road no 639,

Shanghai, People's Republic of China
}

(c) The Author(s). 2018 Open Access This article is distributed under the terms of the Creative Commons Attribution 4.0 International License (http://creativecommons.org/licenses/by/4.0/), which permits unrestricted use, distribution, and reproduction in any medium, provided you give appropriate credit to the original author(s) and the source, provide a link to the Creative Commons license, and indicate if changes were made. The Creative Commons Public Domain Dedication waiver (http://creativecommons.org/publicdomain/zero/1.0/) applies to the data made available in this article, unless otherwise stated. 


\section{Background}

Gonadotropin-releasing hormone (GnRH) antagonists have been used to suppress pituitary activity and to prevent premature surges of luteinising hormone (LH) during controlled ovarian stimulation since the 1990s [1]. GnRH antagonist therapy does not produce a flare effect and rapidly suppresses gonadotropins and it can be initiated in the late follicular phase of the menstrual cycle. Thus, GnRH antagonists are considered to be beneficial for poor responders since there is less suppression in the early follicular phase [2]. Previous studies of poor responders have shown that a GnRH antagonist protocol is associated with decreased cycle cancellation and fewer days of gonadotropin stimulation, but the clinical pregnancy outcomes were not significantly different between GnRH antagonist and agonist protocols [3].

Progress in vitrification techniques has allowed reproductive physicians to consider new strategies for using progestin as an alternative to a $\mathrm{GnRH}$ analogue for improving in vitro fertilisation (IVF) [4-8]. Progestin can inhibit the pre-ovulatory LH surge when it is administered during the early part of the cycle before oestrogen priming [9-11]. Progestin also alters pituitary responsiveness to GnRH and gonadotrophin secretion [12, 13]. For more than 50 years, progestin has been widely applied to control ovulation in hormonal contraception [14], and since 2014, its use has been extended to preventing premature ovulation in $\operatorname{IVF}[5,6]$. Our recent studies demonstrated that progestin-primed ovarian stimulation (PPOS) produced a gradually decreasing LH level during ovarian stimulation, with a low incidence of LH surges $(0.15 \%)$ in women with a normal ovarian reserve [6]. PPOS also produces an acceptable pregnancy outcome compared with the conventional short protocol [4-8]. However, currently, there are no data comparing the efficacy and safety of a GnRH antagonist and progestin in blocking LH surges and premature ovulation in poor responders.

GnRH antagonists have a reported failure rate of approximately $0.34-8.0 \%$ for controlling premature $\mathrm{LH}$ surges in women with a normal ovarian reserve [1-3]. An antagonist failure is more likely to occur in women with advanced age, a diminished ovarian reserve and poor response to gonadotropins [15]. Our prospective trial in a population of poor responders showed that ovulation of the dominant follicle was controlled well using progestin administered orally as a surrogate for a GnRH antagonist, with low incidence rates of LH surges and premature ovulation (3.0\%) [8]. Moreover, progestin priming prolonged the follicular phase by one more day, and the diameter of the pre-ovulatory follicle was larger than those of natural cycle patients. These data indicated that progestin treatment significantly suppressed follicular rupture and provided a wide window for oocyte retrieval [8]. Therefore, we assumed that progestin may show some superiority in controlling premature LH surges compared with $\mathrm{GnRH}$ antagonists in poor responders. Thus, we have developed this well-designed large-sample prospective trial to investigate the potential of progestin for poor responders undergoing IVF treatment.

\section{Methods/design}

In this trial, the efficacies of a GnRH antagonist and progestin are being compared in 340 poor responders undergoing IVF through intracytoplasmic sperm injection (ICSI). The participants will be enrolled in Shanghai Ninth People's Hospital affiliated with Shanghai Jiaotong University School of Medicine. The study has been approved by the Institutional Review Board of Shanghai Ninth People's Hospital (2016-198-T142). Informed consent will be obtained from each patient before any study procedure is performed, in accordance with good clinical practice.

This protocol has been written in accordance with the Standard Protocol Items: Recommendations for Interventional Trials (SPIRIT). A SPIRIT checklist is provided in Additional file 1. Any significant modifications to the protocol will require a formal protocol amendment, agreed by the project team and approved by our Institutional Review Board. Minor administrative changes to the protocol will be documented in a memorandum. The study flowchart is shown in Fig. 1.

\section{Participants}

\section{Inclusion criteria}

The following are the inclusion criteria:

1. Women who have a history of infertility $\geq 1$ year

2. Women aged $>22$ and $<42$ years

3. Women with spontaneous menstrual cycles of $21-$ 35 days

4. Women who have at least one of the following indications for IVF or ICSI: tubal factor, male factor, diminished ovarian reserve, endometriosis or unexplained factors

5. Women diagnosed as poor responders according to the Bologna criteria, including at least two of the three following criteria:

a. Advanced age ( $\geq 40$ years) or any other risk factor for poor ovarian response

b. A previous poor response with no more than three oocytes retrieved using the conventional stimulation protocols

c. Abnormal ovarian reserve test results, including bilateral antral follicle counts $<7$ or serum antiMüllerian hormone $<1.1 \mathrm{ng} / \mathrm{ml}$ 


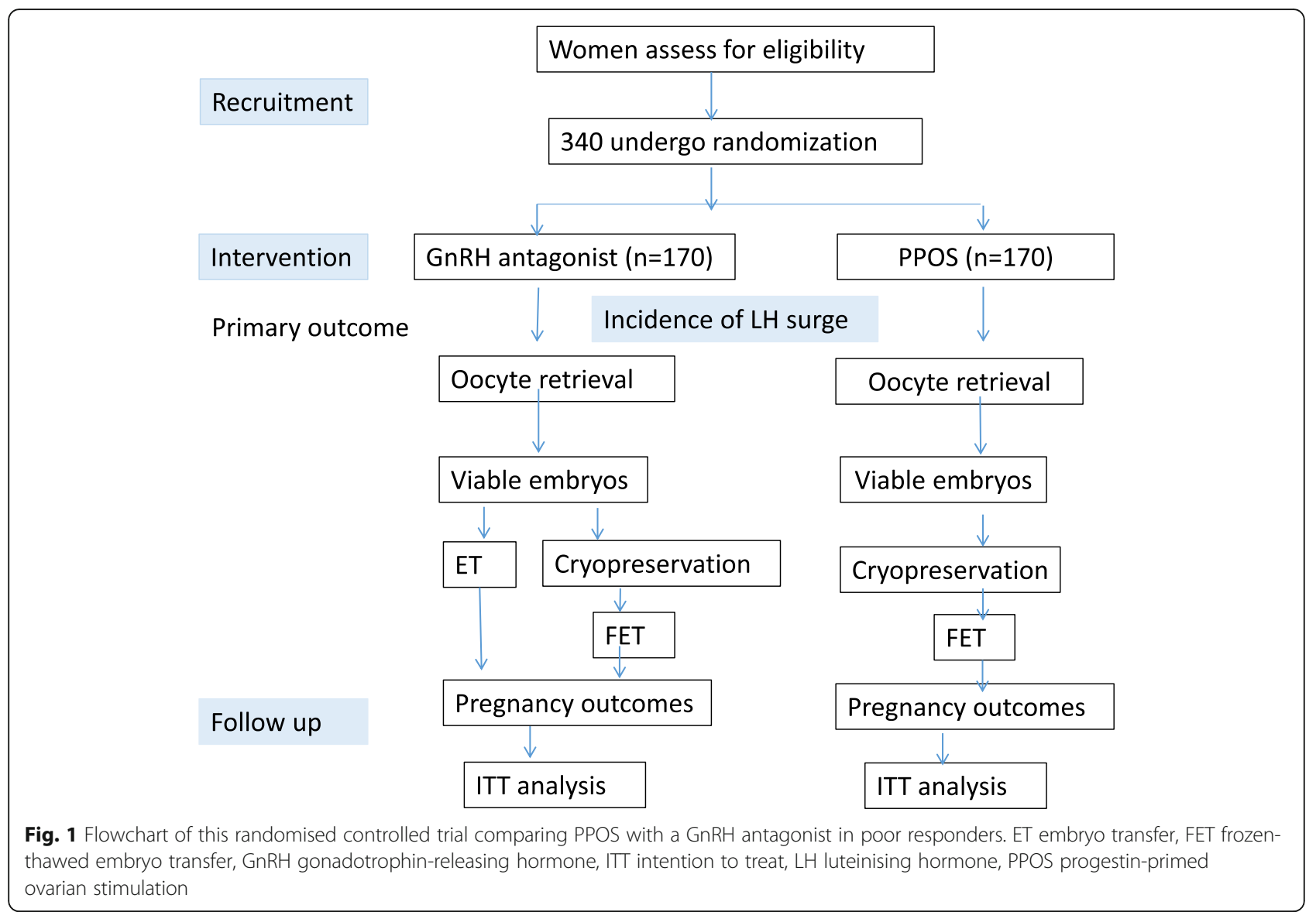

\section{Exclusion criteria}

Women who met any of the following criteria are excluded:

1. Clinically significant systemic diseases, such as renal failure and systemic lupus erythematosus

2. Premature ovarian insufficiency

3. Up to five previous unsuccessful IVF attempts

4. Known Müllerian anomalies

5. Any contraindications to ovarian stimulation treatments

6. Unable to comply with the study procedures

\section{Randomisation}

Participants will be allocated randomly into one of the two arms at a ratio of 1:1 on menstrual cycle day 3. The allocation sequence will be generated utilising computer-generated random numbers. Both investigators and participants will be aware of the allocation after ovarian stimulation. The doctors and embryologists involved in oocyte retrieval and embryo transfer are blinded to the group assignments of the participants in the trial.

\section{Protocols}

\section{GnRH antagonist protocol}

The flexible GnRH antagonist protocol is as follows: 150$225 \mathrm{IU}$ of human menopausal gonadotropin (hMG) is administered daily from menstrual cycle day 3. After 5 days of injections, when the dominant follicles reach a diameter of approximately $14 \mathrm{~mm}, 0.125-0.25 \mathrm{mg}$ of $\mathrm{GnRH}$ antagonist is administered daily up to the trigger day. For women with a low or normal body mass index $(<25.0 \mathrm{~kg} /$ $\mathrm{m}^{2}$ ) or low LH levels before GnRH antagonist administration $(<2.0 \mathrm{mIU} / \mathrm{ml}), 0.125 \mathrm{mg}$ of antagonist is administered daily. For women with a higher body mass index $\left(\geq 25.0 \mathrm{~kg} / \mathrm{m}^{2}\right)$ or LH levels $\geq 2.0 \mathrm{mIU} / \mathrm{ml}, 0.25 \mathrm{mg}$ antagonist will be used daily up to the trigger day. The dose of hMG is adjusted according to the ovarian response. When the dominant follicles reach a diameter of $18 \mathrm{~mm}$, the final stage of oocyte maturation is induced with $100 \mu \mathrm{g}$ of triptorelin via subcutaneous injection and 5000 IU of human chorionic gonadotrophin (hCG) via intramuscular injection. Oocyte retrieval is performed $36 \mathrm{~h}$ later.

\section{PPOS protocol}

hMG at 150-225 IU and medroxyprogesterone acetate (MPA) at $10 \mathrm{mg}$ are administered daily from cycle day 3. 
Five days later, the hMG dose is adjusted according to the ovarian response, while the MPA dose is kept the same up to the trigger day. When the dominant follicles reach a diameter of $18 \mathrm{~mm}$, the final stage of oocyte maturation is induced with $100 \mu \mathrm{g}$ of triptorelin via subcutaneous injection and 5000 IU of hCG via intramuscular injection. Oocyte retrieval is performed $36 \mathrm{~h}$ later.

\section{In vitro fertilisation and embryo culture}

All follicles more than $10 \mathrm{~mm}$ will be retrieved. Follicles are flushed three times at most if no cumulus oocyte complex is present. Standard insemination or ICSI is performed within $6 \mathrm{~h}$ of retrieval. Embryos are examined for the number and regularity of blastomeres and the degree of embryonic fragmentation on the third day. If available, two top-quality embryos (including grade I and grade II, eight-cell blastomere embryos) in the GnRH antagonist group are transferred on the third day. The remaining top-quality embryos are frozen by vitrification, while the non-top-quality embryos are cultured for an extended time. Only blastocysts with good morphology are frozen on day 5 or 6 . All top-quality cleavage embryos and the cryopreserved blastocysts are recorded as viable embryos. In the PPOS group, all top-quality embryos are frozen on the third day, while the non-top-quality embryos are cultured for an extended time and cryopreserved according to the same criteria as above.

\section{Endometrium preparation and frozen-thawed embryo transfer}

Endometrium preparation uses either mild stimulation or hormone replacement therapy. For mild stimulation frozen-thawed embryo transfer cycles, we prescribe letrozole $2.5-5 \mathrm{mg}$ for 3-5 days from cycle day 3 and then monitor follicular growth via serum hormone levels and ultrasound from cycle day 10. At times, treatment includes a low dose of hMG (75 IU/day) to stimulate follicle growth and the endometrial lining. When the diameter of the dominant follicle is $>16 \mathrm{~mm}$ and the endometrial thickness is $>8 \mathrm{~mm}$, with $E_{2}>150 \mathrm{pg} / \mathrm{ml}$ and progesterone $<1.0 \mathrm{ng} / \mathrm{ml}$, one of two procedures is performed, depending upon the LH level. If the serum $\mathrm{LH}$ level is $<20 \mathrm{mIU} / \mathrm{ml}, 5000 \mathrm{IU}$ of hCG is administered at night (21:00) to trigger ovulation, and the transfer of cleavage embryos is arranged for 5 days later. If the $\mathrm{LH}$ level is $>20 \mathrm{mIU} / \mathrm{ml}, 5000 \mathrm{IU}$ of $\mathrm{hCG}$ is injected the same afternoon, and the transfer is conducted 4 days later. The blastocyst transfer is arranged on the sixth or seventh day depending on serum hormone levels and ultrasound results. Dydrogesterone (Abbott Biologicals $\mathrm{BV}$, the Netherlands) was administered orally at $40 \mathrm{mg}$ / day and micronised progesterone capsules at $400 \mathrm{mg} /$ day was administered vaginally for luteal support beginning on the third day after hCG injection.

For patients with a thin endometrium or where the frozen-thawed embryo transfer fails after stimulation cycles, hormone replacement therapy is recommended for endometrial preparation, specifically ethinyl oestradiol administered orally at $75 \mathrm{mcg} /$ day from cycle day 3 onwards, which is commonly used for 14 days. Once the endometrial lining is $>8 \mathrm{~mm}$ thick, Femoston (a yellow tablet) is administered twice per day (Abbott Healthcare Products BV, Weesp, the Netherlands), and $400 \mathrm{mg}$ micronised progesterone capsules are administered daily via an intravaginal route. The time for thawing and transfer is determined on the third day after progesterone administration. The maximum number of transferred embryos is two per cycle. When pregnancy is achieved, the progesterone supplement is continued until 10 weeks of gestation.

\section{Hormonal measurement}

Serum follicle-stimulating hormone (FSH), LH, oestradiol and progesterone levels are monitored during the ovarian stimulation. Hormone levels are measured via chemiluminescence (Abbott Biologicals BV, The Netherlands).

\section{Outcome measurements \\ Primary outcome}

The primary outcome is the incidence of premature LH surges, defined as the serum $\mathrm{LH}>15 \mathrm{mIU} / \mathrm{ml}$ on the trigger day, with or without dominant follicle rupture and increased serum progesterone. Premature ovulation is defined as dominant follicle rupture before the scheduled time. Increased progesterone alone is not defined as the presentation of an LH surge and is listed independently.

\section{Secondary outcomes}

Secondary efficacy parameters include the number of oocytes retrieved, the number of viable embryos, the clinical pregnancy rate, the implantation rate, the ongoing pregnancy rate and the cumulative live birth rates from a single IVF cycle. Clinical pregnancy will be defined as the presence of an intrauterine gestation sac at 7 weeks of gestation. Ongoing pregnancy will be defined as a viable pregnancy at 12 weeks of gestation.

The safety endpoints include the incidence rates of ovarian hyperstimulation syndrome, miscarriage, ectopic pregnancy, pregnancy complication, congenital anomalies and neonatal complications.

\section{Statistics}

\section{Sample size and power calculations}

For the power calculation, previous studies reported that the incidence of premature LH surges in the GnRH antagonist protocol was $8.0 \%$, and our recent data show that the incidence of premature LH surges and ovulation was 3.0\% 
in poor responders using the PPOS protocol. Therefore, we hypothesise that the administration of MPA would decrease the incidence of premature LH surges. The superiority margin is $4.0 \%$. A sample size of 166 in each group would yield $90 \%$ power to establish superiority at the 0.01 level of significance, and 109 in each group yield $90 \%$ power to establish superiority at 0.05 level of significance [16]. Given the abundant clinical resources in our clinic, the number of participants is set as 170 in each group in this trial.

\section{Data management}

The timepoints of enrolment, intervention, data collection and follow-up are described in Fig. 2. Data collected are entered into our electronic data capture system and stored on a secure server at Shanghai Ninth People's Hospital. An automated system for validating data against a set of predefined rules will query investigators regarding data that are invalid, illogical or incomplete. Data elements critical to the primary aim of this trial are double-checked to confirm the accuracy of the data entered compared with the source documents.

\section{Statistical analysis}

We will utilise an intention-to-treat approach with a chi-square test to examine differences in the incidence of premature LH surges. Secondary efficacy parameters and safety parameters will be analysed using a chi-square test for enumeration data and Student's $t$ test for measurement data. $p<0.05$ is considered as a significant difference.

\section{Discussion}

How to control premature LH surges in poor responders has long been an issue in IVF treatment. These poor responders have small quantities of primordial follicle pools and FSH-sensitive follicles, wherein the follicles biologically mature quickly and are prone to premature luteinisation [17]. Therefore, it is more difficult to control premature $\mathrm{LH}$ surges in poor responders than in those with a normal ovarian reserve. $\mathrm{GnRH}$ antagonists accomplish pituitary suppression via a competitive blockage of the $\mathrm{GnRH}$ receptor, but the capability of the endogenous oestrogen-induced $\mathrm{GnRH}$ release is still preserved, and a small proportion of antagonist cycles fail to control LH surges, especially in those of advanced age and with a diminished ovarian reserve [18-20]. In preliminary studies, progestin has been shown to inhibit premature ovulation effectively, and it is useful to compare antagonists and progestin in controlling premature ovulation in poor responders.

To our knowledge, this is the first randomised controlled trial to examine the efficacy of progestin administered orally in blocking LH surges and premature ovulation during ovarian stimulation for poor responders compared with the standard GnRH antagonist protocols. The study results will add to current knowledge on controlled ovarian stimulation and will have the potential to establish a better treatment for poor responders.

\section{Trial status}

The study was conceived and designed in 2016. The registry number is ChiCTR-IPR-17010906 and it was registered on 18 March 2017 (http://www.chictr.org.cn/showproj.aspx?proj=11024). The first participant was randomised on 20 March 2017. We will complete recruitment in July 2018, and patient follow-ups will be ongoing. This protocol, version 2, was approved on 12 January 2017.

\begin{tabular}{|c|c|c|c|c|c|c|c|}
\hline \multirow{3}{*}{ TIMEPOINT } & \multicolumn{7}{|c|}{ Study period } \\
\hline & \multirow[t]{2}{*}{ Enrolment } & \multirow{2}{*}{$\begin{array}{c}\text { Baseline } \\
\text { appointment } \\
\text { No more than } \\
4 \text { weeks }\end{array}$} & \multirow{2}{*}{$\begin{array}{c}\text { Allocation } \\
\text { Menstrual } \\
\text { cycle day } 3\end{array}$} & \multicolumn{4}{|c|}{ Post allocation } \\
\hline & & & & $\begin{array}{l}\text { Cycle } \\
\text { monitor }\end{array}$ & $\begin{array}{l}\text { Oocyte } \\
\text { retrieval }\end{array}$ & $\begin{array}{c}\text { Fresh embryo } \\
\text { transfer }\end{array}$ & $\begin{array}{c}\text { Frozen embryo } \\
\text { transfer }\end{array}$ \\
\hline \multicolumn{8}{|l|}{ ENROLMENT } \\
\hline Identification & $x$ & & & & & & \\
\hline Eligibility screening & $\mathrm{x}$ & & & & & & \\
\hline $\begin{array}{l}\text { Completion of baseline } \\
\text { measures }\end{array}$ & & $x$ & & & & & \\
\hline Informed consent & & $x$ & & & & & \\
\hline Randomisation & & & $x$ & & & & \\
\hline Allocation & & & $x$ & & & & \\
\hline \multicolumn{8}{|l|}{ INTERVENTIONS } \\
\hline GnRH antagonist protocol & & & & $x$ & $x$ & $x$ & $x$ \\
\hline PPOS protocol & & & & $x$ & $x$ & & $x$ \\
\hline \multicolumn{8}{|l|}{ DATA COLLECTION } \\
\hline Demographics & & $x$ & & & & & \\
\hline Primary outcome measure & & & & $x$ & & & \\
\hline Secondary outcome measure & & & & & $x$ & $x$ & $x$ \\
\hline Treatment attendance & & & & \multicolumn{4}{|c|}{$\stackrel{\leftrightarrow}{\longleftrightarrow} \longrightarrow$} \\
\hline Adverse events & & & & \multicolumn{4}{|c|}{$\longleftrightarrow$} \\
\hline \multicolumn{8}{|l|}{ FOLLOW UP } \\
\hline Ongoing pregnancy & & & & & & $x$ & $x$ \\
\hline Live birth & & & & & & $x$ & $x$ \\
\hline
\end{tabular}

Fig. 2 SPIRIT diagram for this protocol comparing PPOS with GnRH antagonist in poor responders. GnRH gonadotrophin-releasing hormone, PPOS progestin-primed ovarian stimulation 


\section{Additional file}

Additional file 1: SPIRIT checklist. (DOC $100 \mathrm{~kb}$ )

\section{Abbreviations}

ET: Embryo transfer; FET: Frozen-thawed embryo transfer; FSH: Folliclestimulating hormone; GnRH: Gonadotrophin-releasing hormone; hCG: Human chorionic gonadotrophin; hMG: Human menopausal gonadotropin; ICSI: Intracytoplasmic sperm injection; ITT: Intention to treat; IVF: In vitro fertilisation; LH: Luteinising hormone; MPA: Medroxyprogesterone acetate; PPOS: Progestin-primed ovarian stimulation

\section{Acknowledgements}

We gratefully acknowledge all staff in the Department of Assisted Reproduction in Shanghai Ninth People's Hospital for their support and cooperation.

\section{Funding}

This study was funded by the National Nature Science Foundation of China (grants 81671520 and 81571397) and the Clinical Research Program of the 9th People's Hospital, Shanghai Jiao Tong University School of Medicine (2016-JYLJ016).

\section{Availability of data and materials}

The final datasets generated and analysed in the current study will be available from the corresponding author on reasonable request. The datasets will be made available from a public repository following the publication of papers related to the relevant data.

\section{Authors' contributions}

YW participated in the design of the study. QC and RC participated in the design and development, including the statistical analysis plan. YK conceived of the study and guided the design. All authors read and approved the final manuscript.

\section{Ethics approval and consent to participate}

Ethical approval has been granted from the Institutional Review Board of Shanghai Ninth People's Hospital. Written consent will be collected from all participants prior to enrolment.

\section{Consent for publication}

Patients will be informed, prior to consenting to participate in the trial, that the results of the study may be presented at academic conferences or published in peer-reviewed journals. Participants will be assured that their confidentiality will be maintained at all times and they will not be identifiable in any publications

\section{Competing interests}

The authors declare that they have no competing interests.

\section{Publisher's Note}

Springer Nature remains neutral with regard to jurisdictional claims in published maps and institutional affiliations.

Received: 3 January 2018 Accepted: 7 August 2018

Published online: 22 August 2018

\section{References}

1. Hall JE, et al. Evidence of differential control of FSH and LH secretion by gonadotropin-releasing hormone $(\mathrm{GnRH})$ from the use of a $\mathrm{GnRH}$ antagonist. J Clin Endocrinol Metab. 1988;67(3):524-31.

2. Al-Inany HG, et al. Gonadotrophin-releasing hormone antagonists for assisted reproductive technology. Cochrane Database Syst Rev. 2016;4: CD001750.

3. Lambalk CB, et al. GnRH antagonist versus long agonist protocols in IVF: a systematic review and meta-analysis accounting for patient type. Hum Reprod Update. 2017;23(5):560-79.

4. Blockeel $\mathrm{C}$, et al. A fresh look at the freeze-all protocol: a SWOT analysis. Hum Reprod. 2016;31(3):491-7.
5. Kuang $Y$, et al. Luteal-phase ovarian stimulation is feasible for producing competent oocytes in women undergoing in vitro fertilization/ intracytoplasmic sperm injection treatment, with optimal pregnancy outcomes in frozen-thawed embryo transfer cycles. Fertil Steril. 2014;101(1): 105-11.

6. Kuang $Y$, et al. Medroxyprogesterone acetate is an effective oral alternative for preventing premature luteinizing hormone surges in women undergoing controlled ovarian hyperstimulation for in vitro fertilization. Fertil Steril. 2015;104(1):62-70.

7. Massin N. New stimulation regimens: endogenous and exogenous progesterone use to block the LH surge during ovarian stimulation for IVF. Hum Reprod Update. 2017;23(1):211-20.

8. Chen $\mathrm{Q}$, et al. Controlled ovulation of the dominant follicle using progestin in minimal stimulation in poor responders. Reprod Biol Endocrinol. 2017;15:71.

9. Dierschke DJ, et al. Blockade by progesterone of estrogen-induced $\mathrm{LH}$ and FSH release in the rhesus monkey. Endocrinology. 1973;92(5):1496-501.

10. Kasa-Vubu JZ, et al. Progesterone blocks the estradiol-induced gonadotropin discharge in the ewe by inhibiting the surge of gonadotropin-releasing hormone. Endocrinology. 1992;131(1):208-12.

11. Richter TA, et al. Progesterone can block the preovulatory gonadotropinreleasing hormone/luteinising hormone surge in the ewe by a direct inhibitory action on oestradiol-responsive cells within the hypothalamus. J Neuroendocrinol. 2005;17(3):161-9.

12. Attardi $B$, et al. Facilitation or inhibition of the oestradiol-induced gonadotrophin surge in the immature female rat by progesterone: effects on pituitary responsiveness to gonadotrophin-releasing hormone $(\mathrm{GnRH})$, $\mathrm{GnRH}$ self-priming and pituitary mRNAs for the progesteronereceptor $\mathrm{A}$ and B isoforms. J Neuroendocrinol. 2007;19(12):988-1000.

13. Soules MR, et al. Progesterone modulation of pulsatile luteinizing hormone secretion in normal women. J Clin Endocrinol Metab. 1984;58(2):378-83.

14. Sitruk-Ware R. New progestagens for contraceptive use. Hum Reprod Update. 2006;12(1):169-78.

15. Reichman DE, et al. Diminished ovarian reserve is the predominant risk factor for gonadotropin-releasing hormone antagonist failure resulting in breakthrough luteinizing hormone surges in in vitro fertilization cycles. Ferti Steril. 2014;102(1):99-102.

16. Chow SC, et al. Sample size calculation in clinical research. 2nd ed. New York: Taylor and Francis; 2007.

17. Wu YG, et al. Aging-related premature luteinization of granulosa cells is avoided by early oocyte retrieval. J Endocrinol. 2015;226(3):167-80.

18. Albano C, et al. Ovarian stimulation with hMG: results of a prospective randomized phase III European study comparing the luteinizing hormonereleasing hormone (LHRH)-antagonist cetrorelix and the $\mathrm{LHRH}$-agonist buserelin. European Cetrorelix Study Group. Hum Reprod. 2000;15(3):526-31.

19. Mansour RT, et al. The use of gonadotropin-releasing hormone antagonist in a flexible protocol: a pilot study. Am J Obstet Gynecol. 2003:189(2):444-6.

20. Messinis IE, et al. Alternate day and daily administration of $\mathrm{GnRH}$ antagonist may prevent premature luteinization to a similar extent during FSH treatment. Hum Reprod. 2005;20(11):3192-7.

\section{Ready to submit your research? Choose BMC and benefit from:}

- fast, convenient online submission

- thorough peer review by experienced researchers in your field

- rapid publication on acceptance

- support for research data, including large and complex data types

- gold Open Access which fosters wider collaboration and increased citations

- maximum visibility for your research: over $100 \mathrm{M}$ website views per year

At $\mathrm{BMC}$, research is always in progress.

Learn more biomedcentral.com/submissions 